Geosciences Journal

pISSN 1226-4806 eISSN 1598-7477

Vol. 20, No. 6, p. 935 - 935, December 2016

DOI 10.1007/s12303-016-0032-y

(c) The Association of Korean Geoscience Societies and Springer 2016

\title{
Erratum to: An application of rock physics modeling to quantify the seismic response of gas hydrate-bearing sediments in Makran accretionary prism, offshore, Pakistan
}

Muhammad Irfan Ehsan* School of Geophysics and information Technology, China University of Geosciences (Beijing), 100083 Beijing, China

Nisar Ahmed

Perveiz Khalid

Institute of Geology, University of the Punjab, Lahore 54590, Pakistan

Liu Xue Wei

School of Geophysics and information Technology, China University of Geosciences (Beijing), 100083 Beijing, China

Mustansar Naeem

Institute of Geology, University of the Punjab, Lahore 54590, Pakistan

Erratum to: Geosciences Journal, 2016, 20, 321-330

DOI 10.1007/s12303-015-0044-z

One of the author names was misprinted. The author list should be corrected as follows.

Muhammed Irfan Ehsan*, Nisar Ahmed, Perveiz Khalid, Liu Xue Wei, and Mustansar Naeem

\section{Corrected Author list}

Muhammad Irfan Ehsan*, Nisar Ahmed, Perveiz Khalid, Liu Xue Wei, and Mustansar Naeem

The online version of the original article can be found under DOI 10.1007/s12303-015-0044-z

*Corresponding author: irfan_opq@yahoo.com 